\title{
Depressive Symptoms in Danish Patients With Glioma and a Cancer-Free Comparison Group
}

\author{
Katrine Løppenthin, $\mathrm{PhD}^{1}$; Christoffer Johansen, MD, DMSc ${ }^{1,2}$; Matilde Bille Larsen, $\mathrm{MScPH}^{3}$; \\ Birgitte Hysse Forchhammer, $\mathrm{PhD}^{4}$; Jannick Brennum, MD, DMSc ${ }^{5}$; Karin Piil, $\mathrm{PhD}^{2}$; Neil Aaronson, $\mathrm{PhD}^{6}$; \\ Birthe Krogh Rasmussen, $\mathrm{MD}, \mathrm{DMSc}^{7}$; and Pernille Bidstrup, $\mathrm{PhD}^{1}$
}

\begin{abstract}
Background: It is well established that patients with glioma may experience adverse general (eg, headache) or focal symptoms (eg, personality changes) and neurocognitive deficits (eg, planning), but they may also experience severe emotional distress. We investigated the prevalence of depressive symptoms in patients with newly diagnosed glioma and in matched cancer-free persons. Methods: For this study, we recruited patients with glioma diagnosed within 12 months at all 4 neurosurgical clinics in Denmark. The cancer-free comparison group was identified through the Danish Central Person Register and matched on sex and age. Participants' depressive symptoms were evaluated using the Center for Epidemiologic Studies Depression Scale (CES-D; score range, 0-60), with a cutoff score $\geq 16$ indicating moderate-to-severe depressive symptoms. Results: In this study, 363 of 554 patients with glioma and 481 of 1,304 cancer-free persons participated. Mean age of all patients was 55 years and $60 \%$ of the population was male. Mean scores for depressive symptoms were statistically significantly higher among patients with glioma, with a mean CES-D score of 10.9 (95\% Cl, 10.1-11.8) compared with 5.3 (95\% Cl, 4.7-5.8) among cancer-free persons $(P<.0001)$. Overall, 92 patients with glioma $(25 \%)$ and 30 cancer-free persons (6\%) had moderate-to-severe depressive symptoms. After adjustment for marital status, education level, and comorbidity, the prevalence of depressive symptoms was 5 times higher among patients with glioma compared with cancer-free persons. Conclusions: A substantially higher prevalence of moderate-tosevere depressive symptoms was identified in patients with glioma compared with cancer-free persons. This indicates the importance of programs to systematically identify and manage depressive symptoms in patients with glioma.
\end{abstract}

J Natl Compr Canc Netw 2020;18(9):1222-1229 doi: $10.6004 /$ jnccn.2020.7570

\footnotetext{
${ }^{1}$ Danish Cancer Society Research Center, Copenhagen; ${ }^{2}$ Department of Oncology, Finsen Center, Rigshospitalet, Copenhagen; ${ }^{3}$ Copenhagen University Hospital GCP Unit, Bispebjerg Hospital, Copenhagen; and ${ }^{4}$ National Study on Young Brain Injury Survivors, Department of Neurology, and ${ }^{5}$ Neurosurgery Clinic, Copenhagen University Hospital Rigshospitalet, Copenhagen, Denmark; ${ }^{6}$ Division of Psychosocial Research and Epidemiology, The Netherlands Cancer Institute, Amsterdam, the Netherlands; and ${ }^{7}$ Department of Neurology, Nordsjællands Hospital, Hillerød, Denmark.
}

\section{Background}

Gliomas are the most common type of primary intracranial tumors in adults, with an incidence rate of 5 per 100,000 and a relative 5-year survival rate of $10 \%{ }^{1}$ Patients experience a significant burden of symptoms related to the disease and its treatment. ${ }^{2,3}$ Frequent symptoms include global cerebral manifestations (fatigue, nausea, headache, and confusion), focal symptoms (hemiparesis, paresthesia, motor control deficiency, visual disturbances, seizures, and speech difficulties), and neurocognitive deficits (impaired attention, concentration difficulty, reduced short-term memory, and personality or behavior changes). 4,5 Treatment depends on the location and size of the tumor, grade of malignancy, and patient age and performance status, ${ }^{6}$ and it often involves a combination of surgery (diagnostic biopsy or surgical resection), ${ }^{7}$ radiotherapy, and chemotherapy. ${ }^{8,9}$ Corticosteroids, analgesics, and antiepileptic treatment are often prescribed for symptom management, contributing to significant adverse effects, such as cognitive dysfunction and fatigue..$^{10}$ Although epidemiologic data, in general, indicate that depression is 2 to 3 times more prevalent in patients with cancer than in the general population, a meta-analysis of 211 studies of patients with cancer showed that patients with brain tumors had the highest prevalence of self-reported depression. ${ }^{11}$ Furthermore, authors of a systematic review of $42 \mathrm{ob}$ servational studies reported that a median of $27 \%$ of adults with glioma had depression; however, the prevalence ranged widely, depending on the study design and diagnostic methods used. ${ }^{12}$ Furthermore, important methodological limitations exist in these studies, including small sample sizes, which compromise precise estimation of the prevalence, and, most important, the absence of a control group. Such information is important because depression may have a severe impact on

See JNCCN.org for supplemental online content. 
patient outcomes, including neurologic function and performance status, ${ }^{13}$ impaired health-related quality of life, ${ }^{14,15}$ and even survival. ${ }^{16,17}$ In the present study, we investigated the prevalence of self-reported moderateto-severe depressive symptoms in patients with glioma and compared the prevalence with that in a sex- and agematched cancer-free comparison group derived from the general population. We also investigated associations between various clinical factors and moderate or severe self-reported depressive symptoms in patients with glioma.

\section{Methods}

We used data collected for the Danish part of the international GLIOGENE (Glioma Gene) study, ${ }^{18}$ which addressed epidemiologic and biologic risk factors for the development of glioma. Thus, the inclusion criteria were developed for this international study. All patients aged 18-80 years with a primary low- or high-grade glioma (ICD-O-3 morphology codes 94303, 94013, 94003, 94203, 94113, 94103, 94003, 93913, 93923, 93941, 93913, 94413, 94403, 93803, 93803, 93813, 94423, 93823, 93853, 94503, 94513, 93823, 94243, and 93831) diagnosed at 1 of the 4 neurosurgical clinics in Denmark between June 15, 2001, and December 31, 2013, were invited to participate in the study. The diagnosis had to have been made no more than 12 months before entry into the study. We excluded individuals who were unable to read or understand Danish and those with severely low cognitive function, who were thus potentially unable to cooperate or give informed consent. No formal evaluation of the patients' cognition was performed, but patients were subjectively evaluated by the interviewer.

Cancer-free persons were identified through the Danish Central Person Register ${ }^{19}$ and matched on sex and age in 5-year intervals as control subjects (ratio of 2 controls per glioma patient). The comparison group consisted of cancer-free persons; however, we had no information on whether these persons had other illnesses. The cancer-free persons were invited to participate between June 15, 2001, and December 31, 2013, as were the patients with glioma. All participants were contacted either by a nurse at 1 of the 4 neurosurgical clinics or by mail and invited to participate in an interview to determine depressive symptoms. The interviews were conducted by a nurse research coordinator and took place at one of the neurosurgical clinics, at the person's home, or at the Danish Cancer Society Research Center. All participants provided written consent. The study protocol was approved by the Danish National Committee on Health Research Ethics (H-2-2010-032) and the Danish Data Protection Agency (2013-41-1502).

\section{Outcome Measures}

Depressive symptoms were measured using the Center for Epidemiologic Studies Depression Scale (CES-D), ${ }^{20}$ which was administered during an interview conducted by a trained nurse. The patients indicated how often during the previous week they had experienced each of 20 symptoms on a scale from 0 to $3(0=$ rarely or none of the time; 1 = some or a little of the time; 2 = occasionally or a moderate length of time; $3=$ most or all the time). The scores range from 0 to 60 , with a cutoff of $\geq 16$ indicating moderate-to-severe depressive symptoms. ${ }^{20}$

Age at diagnosis, sex (male or female), marital status (married/living with a partner vs divorced/single), education level (low, $\leq 10$ years of schooling; medium, 11-12 years of schooling; high, $\geq 13$ years of schooling), and comorbidities (absent or present, including diabetes mellitus, emphysema, chronic bronchitis, chronic obstructive pulmonary disease, rheumatoid arthritis, lupus, liver diseases, AIDS/HIV, stomach ulcer, hepatitis, heart attack, stroke, and cerebral blood clot) were ascertained at interviews. Date of diagnosis, tumor type, stage, and morphology (grades 1 and 2, 3, and 4) were obtained from the patients' medical records.

\section{Statistical Analyses}

To examine the generalizability of the study results, we compared the age and sex of the patients with glioma who participated in the study with the age and sex of those who did not. In addition, we compared the age and sex of the cancer-free persons who participated in the study with the cancer-free persons who were invited but did not participate.

We used descriptive statistics (means, SDs, and frequencies) to characterize the patients with glioma and the cancer-free persons, and using a $t$ test we compared the average depressive symptom scores of the 2 groups. Although age and sex were the only matching criteria for the original study on glioma risk, we adjusted for marital status, education level, and comorbidity ${ }^{21,22}$ in the logistic regression models because they may act as potential confounders. In these analyses, we compared the prevalence of moderate-to-severe depressive symptoms in patients with glioma and in cancer-free persons; results are presented as odds ratios (ORs) with $95 \%$ CIs, and a $P$ value $<.01$ was considered to be statistically significant. In descriptive analyses (numbers and frequencies), we compared the sex, age, education level, marital status, presence of comorbidity, tumor grade, and time since diagnosis of patients with glioma with moderate-tosevere depressive symptoms versus those with no depressive symptoms. We calculated differences in mean depressive score between 3 periods after glioma diagnosis ( $\leq 3,>3$ to $\leq 6$, and $>6$ months) and compared with the cancer-free persons and presented them as means (SDs), $\beta$-estimates, and significance levels. All statistical analyses were performed using SAS 9.3 software (SAS Institute Inc). 


\section{Results}

A total of 554 patients with glioma and 1,304 cancer-free persons fulfilled the inclusion criteria and were invited to participate in the study. Of these, 15 patients with glioma and 261 of the cancer-free persons were not reached, 72 with glioma and 471 cancer-free persons did not agree to participate, and 6 patients with glioma and 28 cancerfree persons were excluded for unknown reasons. We excluded participants with missing data for the CES-D (98 patients with glioma and 63 cancer-free persons). This resulted in 363 patients with glioma (response rate, $66 \%$ ) and 481 cancer-free persons (response rate, $37 \%$ ) included in this study (Figure 1). On average, the patients with glioma were interviewed 4 months after diagnosis.

We found no significant differences in age, sex, or tumor grade between the patients with glioma who participated in the study and those who did not, and no significant differences were seen in age or sex between the cancer-free persons who participated and those who were invited but refused to participate or for whom data were missing (supplemental eTable 1, available with this article at JNCCN.org).

\section{Study Cohort Characteristics}

Characteristics of the study samples are presented in Table 1. Men represented $60 \%$ of the participants,

A

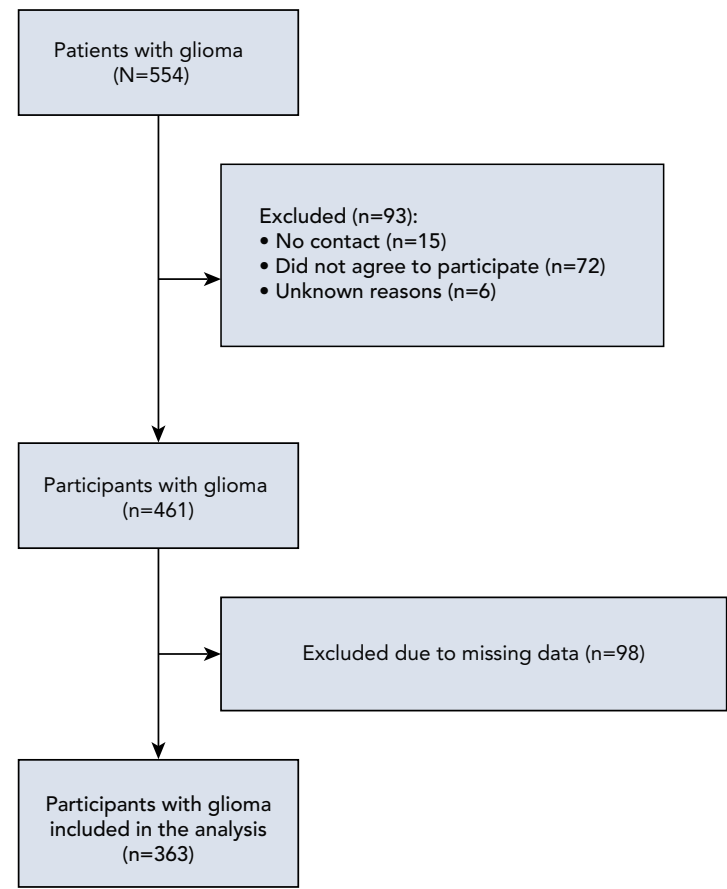

and the average age was 55 years (SD, 13.4). Among the patients with glioma, $60 \%$ were diagnosed with glioblastoma.

\section{Severity of Depressive Symptoms}

In total, 92 patients with glioma had moderate-to-severe depressive symptoms (CES-D score, $\geq 16$ ). Patients with moderate-to-severe depressive symptoms had a mean age of 56 years; $53 \%$ were male; most $(77 \%)$ were living with a partner; $66 \%$ had tumor grade 4 ; and half of the patients had been diagnosed within the past 3 months.

The average depressive symptom scores were 10.8 (95\% CI, 10.1-11.8) in the group of patients with glioma and $5.3(95 \% \mathrm{CI}, 4.7-5.8)$ in the cancer-free persons $(P<.0001)$. Moderate-to-severe depressive symptoms were present in $25 \%$ of the patients with glioma and $6 \%$ of the cancer-free comparison group $(P<.0001)$ (Table 1$)$.

After adjustment for age and sex, we found an increased prevalence of depressive symptoms in patients with glioma, with an OR of 5.16 (95\% CI, 3.32-8.01) compared with cancer-free persons. After further adjustment for sex, age, marital status, education level, and comorbidity, the prevalence of depressive symptoms remained statistically significantly higher for patients with glioma, with an OR of 5.12 (95\% CI, 3.29-7.98) compared with cancer-free persons. In a comparison of

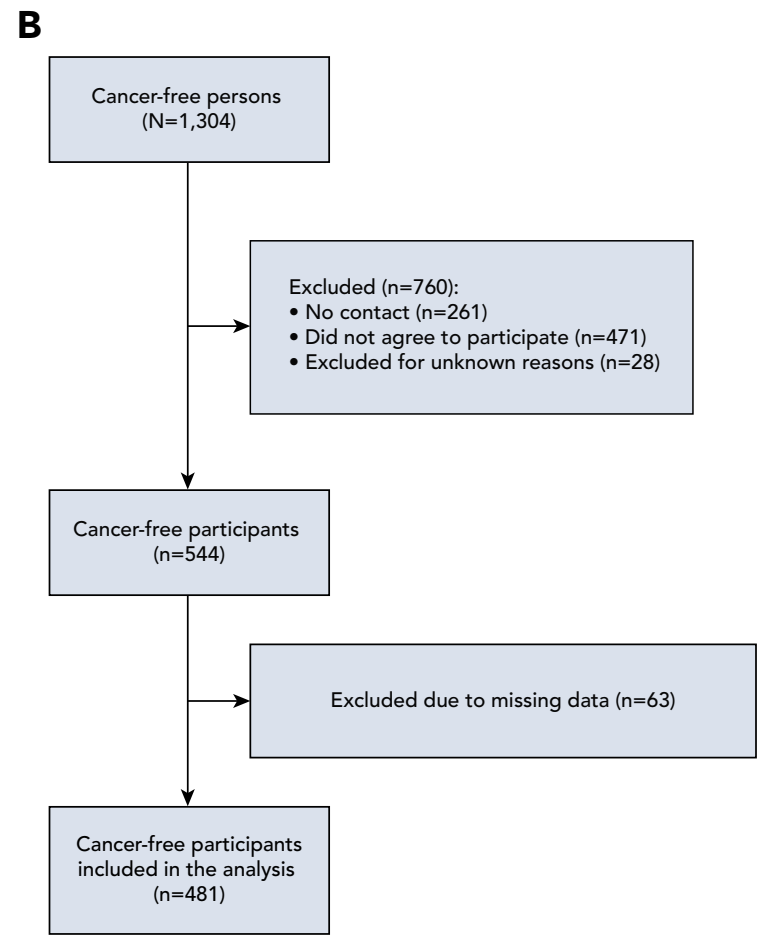

Figure 1. Cohort selection for (A) patients with glioma and (B) cancer-free persons. 


\section{Table 1. Demographic and Clinical Characteristics}

\begin{tabular}{|c|c|c|c|}
\hline & $\begin{array}{c}\text { Patients With Glioma } \\
\text { n (\%) }\end{array}$ & $\begin{array}{c}\text { Cancer-Free Persons } \\
\text { n (\%) }\end{array}$ & $P$ Value \\
\hline Sex & & & .83 \\
\hline Male & $220(61)$ & $288(60)$ & \\
\hline Female & $143(39)$ & $193(40)$ & \\
\hline Age, mean (SD), y & $55.4(13)$ & $55.2(14)$ & .80 \\
\hline Depressive symptoms score, mean (SD) & $10.9(8)$ & $5.3(6)$ & $<.0001$ \\
\hline Moderate-to-severe depressive symptoms (CES-D score, $\geq 16$ ) & $92(25)$ & $30(6)$ & \multirow{2}{*}{$<.0001$} \\
\hline Low depressive symptoms (CES-D score, $<16$ ) & $271(75)$ & $451(94)$ & \\
\hline Education levela & & & .04 \\
\hline Low & $64(18)$ & $63(13)$ & \\
\hline Medium & $187(52)$ & $233(48)$ & \\
\hline High & $112(31)$ & $185(39)$ & \\
\hline Marital status & & & .73 \\
\hline Married/Living with a partner & $288(79)$ & $377(78)$ & \\
\hline Divorced/Single & $75(21)$ & $104(22)$ & \\
\hline Comorbidities & & & .94 \\
\hline Absent $(0)$ & $255(70)$ & $339(71)$ & \\
\hline Present $(\geq 1)$ & $108(30)$ & $142(30)$ & \\
\hline \multicolumn{4}{|l|}{ Tumor grade } \\
\hline 1 and 2 & $79(22)$ & & \\
\hline 3 & $57(16)$ & & \\
\hline 4 & $225(62)$ & & \\
\hline \multicolumn{4}{|l|}{ Tumor type } \\
\hline Astrocytoma, diffuse & $34(9)$ & & \\
\hline Astrocytoma, anaplastic & $26(7)$ & & \\
\hline Ependymoma & $5(1)$ & & \\
\hline Glioblastoma & $218(60)$ & & \\
\hline Glioma, unclassified anaplastic & $6(2)$ & & \\
\hline Gliosarcoma & $7(2)$ & & \\
\hline Oligoastrocytoma & $9(3)$ & & \\
\hline Oligoastrocytoma, anaplastic & $3(1)$ & & \\
\hline Oligodendroglioma & $28(8)$ & & \\
\hline Oligodendroglioma, anaplastic & $22(6)$ & & \\
\hline Pleomorphic xanthoastrocytoma & $1(0.3)$ & & \\
\hline Subependymoma & $2(1)$ & & \\
\hline Time since diagnosis, mean (SD), mo & $4.18(3)$ & & \\
\hline$\leq 3 \mathrm{mo}$ & $179(50)$ & & \\
\hline$>3$ to $\leq 6 \mathrm{mo}$ & $110(30)$ & & \\
\hline$>6 \mathrm{mo}$ & $72(20)$ & & \\
\hline
\end{tabular}

Abbreviation: CES-D, Center for Epidemiologic Studies Depression Scale.

a Low, $\leq 10$ years of schooling; medium, $11-12$ years of schooling; high, $\geq 13$ years of schooling

patients with glioma with moderate-to-severe (CES-D score, $\geq 16$ ) and low depressive symptom scores (CES-D score, $<16$ ), we found no statistically significant difference according to sex, age, comorbidity, or tumor grade (Table 2). Compared with cancer-free persons, only patients diagnosed with glioma $>6$ months after diagnosis had 


\begin{tabular}{|c|c|c|c|}
\hline & $\begin{array}{c}\text { Moderate-to-Severe Depressive Symptoms } \\
\text { (CES-D Score } \geq 16 \text { ) } \\
n(\%)\end{array}$ & $\begin{array}{l}\text { No Depressive Symptoms } \\
\text { (CES-D Score <16) } \\
\text { n (\%) }\end{array}$ & $P$ Value \\
\hline Total, n & 92 & 269 & \\
\hline Sex & & & .08 \\
\hline Male & $49(53)$ & $171(64)$ & \\
\hline Female & $43(47)$ & $98(36)$ & \\
\hline Age, mean (SD), y & $55.7(14)$ & $55.3(13)$ & .81 \\
\hline Education levela & & & .53 \\
\hline Low & $15(16)$ & $49(18)$ & \\
\hline Medium & $52(57)$ & $134(50)$ & \\
\hline High & $25(27)$ & $86(32)$ & \\
\hline Marital status & & & .47 \\
\hline Married/Living with a partner & 71 (77) & $217(81)$ & \\
\hline Divorced/Single & $21(23)$ & $52(19)$ & \\
\hline Comorbidities & & & .46 \\
\hline Absent (0) & $57(62)$ & $178(66)$ & \\
\hline Present $(\geq 1)$ & $35(38)$ & $91(34)$ & \\
\hline Tumor grade & & & .48 \\
\hline 1 and 2 & $16(17)$ & $63(23)$ & \\
\hline 3 & $15(16)$ & $42(16)$ & \\
\hline 4 & $61(66)$ & $164(61)$ & \\
\hline Time since diagnosis, mo & & & .54 \\
\hline$\leq 3$ & $46(50)$ & $133(49)$ & \\
\hline$>3$ to $\leq 6$ & $31(34)$ & $79(29)$ & \\
\hline$>6$ & $15(16)$ & $57(21)$ & \\
\hline
\end{tabular}

Abbreviation: CES-D, Center for Epidemiologic Studies Depression Scale.

aLow, $\leq 10$ years of schooling; medium, 11-12 years of schooling; high, $\geq 13$ years of schooling.

significantly increased depressive symptoms ( -4.13 points; $95 \%$ CI, -5.83 to -2.43 ) (Table 3 ).

\section{Discussion}

We observed a 5 times higher prevalence of moderate-tosevere depressive symptoms in Danish patients with glioma than in cancer-free persons. The highest scores for depressive symptoms were observed 3 to 6 months after diagnosis; although they decreased subsequently, the scores remained significantly higher than in the cancer-free persons. This finding is in line with previous longitudinal studies reporting a higher incidence of depressive symptoms during the first year after diagnosis. ${ }^{23,24}$ In a longitudinal study of 30 patients with high-grade glioma, depressive symptoms were highest at baseline (1 week after surgery) and lowest after week $52,{ }^{23}$ but with a

Table 3. Depressive Symptoms at 3 Time Points After Glioma Diagnosis ${ }^{a}$

\begin{tabular}{|lccc|}
\hline & Mean (SD) & $\boldsymbol{\beta}$-Estimate $(\mathbf{9 5} \%$ Cl) & $\boldsymbol{P}$ Value \\
\hline Time since diagnosis $\leq 3 \mathrm{mo}(\mathrm{n}=179)$ & $11.3(8.2)$ & $1.69(-0.2 \text { to } 3.57)^{\mathrm{b}}$ & $2.10(0.04 \text { to } 4.15)^{\mathrm{b}}$ \\
\hline Time since diagnosis $>3$ mo and $\leq 6 \mathrm{mo}(\mathrm{n}=110)$ & $11.7(8.8)$ & 0.0 & .05 \\
\hline Time since diagnosis $>6$ mo $(\mathrm{n}=74)$ & $9.5(8.5)$ & $-4.13(-5.83 \text { to }-2.43)^{\mathrm{c}}$ & $<.0001$ \\
\hline Cancer-free control subjects $(\mathrm{n}=481)$ & $5.3(5.9)$ &
\end{tabular}

aAdjusted for sex, age, marital status, education level, and comorbidity.

${ }^{b}$ Comparison between the 3 time periods.

'Comparison between each of the 3 periods and cancer-free persons. 
nonsignificant decrease in depressive symptoms over time. Thus, despite reductions in depressive symptoms over time, it cannot be assumed that patients with glioma will recover from these symptoms on their own.

As in previous studies, ${ }^{25,26}$ we did not detect statistically significant differences in patient-related factors (sociodemographic characteristics) or clinical factors (comorbidity, tumor extent, and disease duration) among patients with low versus moderate-to-severe depressive symptoms. A longitudinal multicenter study of 598 patients with high-grade glioma found, however, that patients with multifocal tumor sites and large tumors more frequently experienced postsurgery depressive symptoms than patients with a tumor at one site or with small tumors. ${ }^{13}$ The study by Litofsky et al ${ }^{13}$ only included patients with high-grade glioma and measured depressive symptoms after surgery using 36-Item Short Form Health Survey mental health summary scores, whereas in the present study, we included both low- and high-grade glioma and measured depressive symptoms at different time points using the CES-D. These differences make direct comparison between the studies difficult; however, the study by Litofsky et al ${ }^{13}$ does indicate that the impact of tumor site and size on depressive symptoms needs further investigation.

Although no evidence exists, it is possible that the explanation for the development of depressive symptoms in patients with glioma includes clinical factors. Although neither chemotherapy nor radiotherapy has been associated with depressive symptoms in patients with glioma, ${ }^{12}$ the commonly used treatment with glucocorticoids has been associated with depressive symptoms. ${ }^{13}$ This may be due to the fact that glucocorticoids are prescribed primarily for large tumors and poor disease status, which may contribute to mood disturbances, ${ }^{27}$ or to the direct adverse effects of glucocorticoids, including well-known symptoms of depression such as cognitive dysfunction, sleep disturbance, and fatigue. ${ }^{28}$ Biochemical changes or a direct destructive effect of the glioma may also act as a potential explanatory biologic mechanism, because they can cause specific symptoms such as learning and memory impairment and concentration problems, which are also symptoms of depression. ${ }^{2,29}$ The existential reaction to the disease and the poor prognosis may also play a role. Although the short life expectancy of patients with highgrade glioma makes them vulnerable to developing depressive symptoms, ${ }^{30}$ existential concerns are likely to play a role for patients with low-grade glioma, including anxiety arising when a brain tumor is diagnosed and related to treatment and its effectiveness, adverse effects of antiepileptic drugs, and uncertainty about family and work. ${ }^{30}$

\section{Clinical Implications}

Failure to recognize severe depressive symptoms may delay or even prevent patients from receiving appropriate treatment. According to the ICD- $10^{31}$ for depression, moderate-to-severe depressive symptoms can pervade various aspects of life, including inability to experience joy, feelings of worthlessness, withdrawal from social relationships, irritability, insomnia, and symptoms of psychosis, including delusions or hallucinations and suicidality. A systematic review and metaanalysis documented worse survival for patients with both low- and high-grade glioma with depression, regardless of time of diagnosis, ${ }^{32}$ which underlines the importance of identifying and treating depression in this patient group.

Thus, in view of the high prevalence of depressive symptoms in patients with glioma, treatment (antidepressants and psychotherapy, such as cognitive behavioral therapy) may be particularly relevant. A metaanalysis $^{33}$ of 563 patients with primary breast cancer showed that depressive symptoms can be treated effectively with antidepressants, with an estimated effect size of 1.56 (95\% CI, 1.07-2.28) compared with placebo. Walker et $\mathrm{al}^{34}$ concluded, in a systematic review of 7 randomized controlled trials, that there is some evidence for the effectiveness of antidepressants alone or in combination with psychologic treatment in patients with cancer with depression. However, we still lack knowledge of the effects of psychologic counseling and antidepressant treatment specifically in patients with glioma. Better understanding of these effects can contribute to clinical guidelines for the prevention and treatment of depressive symptoms in patients with glioma. Furthermore, future studies could benefit from including more information on clinical factors that may contribute to depressive symptoms in patients with glioma. Such information may contribute to identification of patients with glioma at high risk of developing depression.

\section{Study Limitations}

The cross-sectional design of this study did not allow determination of causal directions. Although $80 \%$ of the patients completed the CES-D within 6 months of diagnosis, variation in the time since diagnosis might have influenced the prevalence of reported depressive symptoms. We had no information on whether the patients with glioma or the cancer-free persons were receiving antidepressant treatment, and we may therefore have underestimated the prevalence of moderate-tosevere depressive symptoms. Furthermore, we had no information about other psychologic symptoms, such as anxiety or posttraumatic stress disorder, which could have influenced the depressive symptoms..$^{35,36}$ Although we included clinical factors, we had no information on 
tumor site or size or the treatment received, restricting our ability to examine these potential risk factors. Exclusion of patients aged $>80$ years limits the ability to generalize the results to the oldest age group of patients with glioma, in whom depressive symptoms may be overlooked. In addition, the exclusion of patients with severely decreased cognitive function limited our ability to cover the entire population of patients with glioma and limited the generalizability of our results to patients with no or only limited cognitive problems; this may have resulted in underestimation of the prevalence of depressive symptoms in the population of patients with glioma. Furthermore, this leaves open the question of how depressive symptoms can best be measured in patients who may have difficulty participating in questionnaire studies or even in psychiatric interviews to evaluate depressive symptoms. Of importance is also the fact that patients diagnosed with high tumor grades were less likely to agree to participate in the study, which may also impact the reported prevalence of depressive symptoms and calls for further studies to examine depressive symptoms in patients with high-grade tumors. Finally, the response rate of the cancer-free population was quite low (37\%), which may limit the generalizability to the cancer-free population.

A strength of this study is greater robustness when assessing the prevalence of depressive symptoms in patients with glioma, because our study included a sexand age-matched cancer-free comparison group, was nationwide, and covered one of the largest populations studied so far. The inclusion of cancer-free persons identified through the Danish Central Person Register is a major strength of the study, because it allowed a comparison level of depressive symptoms in persons of similar age and sex, indicating the added burden of a glioma diagnosis. Most previous studies had either no comparison group or a comparison group consisting of the spouses of patients with glioma, who have been reported to have levels of depressive symptoms similar to or even higher than those of patients with glioma. ${ }^{37}$ Inclusion of several glioma-specific clinical factors in our study also made it possible to evaluate the relationship of these factors to depressive symptoms.

\section{Conclusions}

In one of the first studies to include a cancer-free comparison group, we found a 5 times higher prevalence of moderate-to-severe depressive symptoms in patients with glioma than in cancer-free persons. The increased prevalence of depressive symptoms was not explained by differences in age, sex, tumor type, or comorbidity. Regular screening for depressive symptoms in patients with glioma might help to identify those who require further psychiatric assessment and treatment of clinical depression.

\footnotetext{
Submitted July 30, 2019; accepted for publication April 1, 2020.
}

Author contributions: Study design: Løppenthin, Johansen, Larsen, Bidstrup. Data collection and interpretation: Løppenthin, Johansen, Larsen, Bidstrup. Interpretation of results: All authors. Manuscript editing: All authors. Approval of final manuscript: All authors.

Disclosures: The authors have disclosed that they have not received any financial consideration from any person or organization to support the preparation, analysis, results, or discussion of this article.

Correspondence: Katrine Løppenthin, PhD, Danish Cancer Society Research Center, Strandboulevarden 49, DK-2100 Copenhagen, Denmark. Email: katrine.bjerre.loeppenthin@regionh.dk

\section{References}

1. Stupp R, Hegi ME, Mason WP, et al. Effects of radiotherapy with concomitant and adjuvant temozolomide versus radiotherapy alone on survival in glioblastoma in a randomised phase III study: 5-year analysis of the EORTC-NCIC trial. Lancet Oncol 2009;10:459-466.

2. Armstrong TS, Cohen MZ, Eriksen LR, Hickey JV. Symptom clusters in oncology patients and implications for symptom research in people with primary brain tumors. J Nurs Scholarsh 2004;36:197-206.

3. Kim S. A longitudinal study of lipid peroxidation and symptom clusters in patients with brain cancers. Nurs Res 2018;67:387-394

4. Piil K, Jakobsen J, Christensen KB, et al. Needs and preferences among patients with high-grade glioma and their caregivers - a longitudinal mixed methods study. Eur J Cancer Care (Engl) 2018;27: e12806.

5. Coomans MB, Dirven L, Aaronson NK, et al. Symptom clusters in newly diagnosed glioma patients: which symptom clusters are independently associated with functioning and global health status? Neuro Oncol 2019; 21:1447-1457.

6. Weller M, van den Bent M, Hopkins K, et al. EANO guideline for the diagnosis and treatment of anaplastic gliomas and glioblastoma. Lancet Oncol 2014;15:e395-403.

7. Khasraw M, Lassman AB. Advances in the treatment of malignant gliomas. Curr Oncol Rep 2010;12:26-33.

8. Shaw $E$, Arusell $R$, Scheithauer B, et al. Prospective randomized trial of low- versus high-dose radiation therapy in adults with supratentorial

low-grade glioma: initial report of a North Central Cancer Treatment Group/Radiation Therapy Oncology Group/Eastern Cooperative Oncology Group study. J Clin Oncol 2002;20:2267-2276.

9. Shaw EG, Wang M, Coons SW, et al. Randomized trial of radiation therapy plus procarbazine, lomustine, and vincristine chemotherapy for supratentorial adult low-grade glioma: initial results of RTOG 9802. J Clin Oncol 2012;30:3065-3070.

10. Deutsch MB, Panageas KS, Lassman $A B$, et al. Steroid management in newly diagnosed glioblastoma. J Neurooncol 2013;113:111-116.

11. Krebber AM, Buffart LM, Kleijn G, et al. Prevalence of depression in cancer patients: a meta-analysis of diagnostic interviews and self-report instruments. Psychooncology 2014;23:121-130.

12. Rooney AG, Carson A, Grant R. Depression in cerebral glioma patients: a systematic review of observational studies. J Natl Cancer Inst 2011;103: 61-76.

13. Litofsky NS, Farace $E$, Anderson F Jr, et al. Depression in patients with high-grade glioma: results of the Glioma Outcomes Project. Neurosurgery 2004;54:358-367.

14. Pelletier G, Verhoef MJ, Khatri N, et al. Quality of life in brain tumor patients: the relative contributions of depression, fatigue, emotional distress, and existential issues. J Neurooncol 2002;57:41-49.

15. Mainio A, Hakko H, Niemelä A, et al. Gender difference in relation to depression and quality of life among patients with a primary brain tumor. Eur Psychiatry 2006;21:194-199. 
16. Mainio A, Hakko H, Niemelä A, et al. Depression and functional outcome in patients with brain tumors: a population-based 1-year follow-up study. J Neurosurg 2005;103:841-847.

17. Gathinji M, McGirt MJ, Attenello FJ, et al. Association of preoperative depression and survival after resection of malignant brain astrocytoma. Surg Neurol 2009;71:299-303.

18. Melin BS, Barnholtz-Sloan JS, Wrensch MR, et al. Genome-wide association study of glioma subtypes identifies specific differences in genetic susceptibility to glioblastoma and non-glioblastoma tumors [letter]. Nat Genet 2017;49:789-794.

19. Pedersen CB. The Danish Civil Registration System. Scand J Public Health 2011;39(7 Suppl):22-25.

20. Radloff LS. The CES-D scale: a self-report depression scale for research in the general population. Appl Psychol Meas 1977;1:385-401.

21. Niedzwiedz CL, Knifton $L$, Robb KA, et al. Depression and anxiety among people living with and beyond cancer: a growing clinical and research priority. BMC Cancer 2019;19:943.

22. Arnold SD, Forman LM, Brigidi BD, et al. Evaluation and characterization of generalized anxiety and depression in patients with primary brain tumors. Neuro-oncol 2008;10:171-181.

23. Piil K, Jakobsen J, Christensen KB, et al. Health-related quality of life in patients with high-grade gliomas: a quantitative longitudinal study. J Neurooncol 2015;124:185-195.

24. Rooney AG, McNamara S, Mackinnon M, et al. Frequency, clinical associations, and longitudinal course of major depressive disorder in adults with cerebral glioma. J Clin Oncol 2011;29:4307-4312.

25. Madhusoodanan S, Danan D, Moise D. Psychiatric manifestations of brain tumors: diagnostic implications. Expert Rev Neurother 2007;7: 343-349

26. Mainio A, Hakko $H$, Niemelä A, et al. Depression in relation to anxiety, obsessionality and phobia among neurosurgical patients with a primary brain tumor: a 1-year follow-up study. Clin Neurol Neurosurg 2011;113 649-653.

27. Acquaye AA, Vera-Bolanos E, Armstrong TS, et al. Mood disturbance in glioma patients. J Neurooncol 2013;113:505-512

28. Wen PY, Kesari S. Malignant gliomas in adults. N Engl J Med 2008;359: 492-507.

29. Clark L, Chamberlain SR, Sahakian BJ, Neurocognitive mechanisms in depression: implications for treatment. Annu Rev Neurosci 2009;32:57-74.

30. Kilbride L, Smith G, Grant R. The frequency and cause of anxiety and depression amongst patients with malignant brain tumours between surgery and radiotherapy. J Neurooncol 2007;84:297-304.

31. World Health Organization (WHO). International Statistical Classification of Diseases and Related Health Problems. 10th Revision. Accessed July 17, 2019. Available at: https://icd.who.int/browse10/2016/en

32. Shi C, Lamba N, Zheng LJ, et al. Depression and survival of glioma patients: a systematic review and meta-analysis. Clin Neurol Neurosurg 2018;172:8-19

33. Laoutidis ZG, Mathiak K. Antidepressants in the treatment of depression depressive symptoms in cancer patients: a systematic review and metaanalysis. BMC Psychiatry 2013;13:140

34. Walker J, Sawhney A, Hansen $\mathrm{CH}$, et al. Treatment of depression in adults with cancer: a systematic review of randomized controlled trials. Psycho Med 2014:44:897-907.

35. Shalev A, Liberzon I, Marmar C. Post-traumatic stress disorder. N Engl J Med 2017;376:2459-2469.

36. Tiller JW. Depression and anxiety. Med J Aust 2013;199(Suppl 6):S28-31

37. Goebel S, von Harscher M, Mehdorn HM. Comorbid mental disorders and psychosocial distress in patients with brain tumours and their spouses in the early treatment phase. Support Care Cancer 2011;19: 1797-1805.

\section{ENROLL NOW}

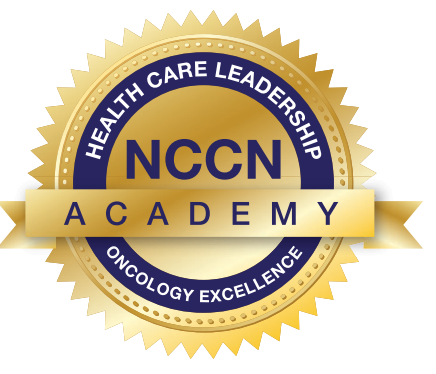

\section{NCCN Academy for Excellence \& Leadership in Oncology ${ }^{\mathrm{TM}}$}

\section{School of Pharmaceutical \& Biotech Business}

\section{VIRTUAL PROGRAM}

Tuesday, October 6 | 12:30 - 3:30 PM ET

Module I: Oncology Medical Home: Perspectives on Evolution, Impact \& Future State

\section{Module II: Pathways: A View Across Developers and Stakeholders}

This program provides pharmaceutical and biotechnology professionals with the significant opportunity to view the oncology space and its current and future operational issues from providers, payers, patient advocates, and other stakeholders.

\section{Enroll Today: NCCN.org/academy/virtual-october}


Supplemental online content for:

\section{Depressive Symptoms in Danish Patients With Glioma and a Cancer-Free Comparison Group}

Katrine Løppenthin, PhD; Christoffer Johansen, MD, DMSc; Matilde Bille Larsen, MScPH;

Birgitte Hysse Forchhammer, PhD; Jannick Brennum, MD, DMSc; Karin Piil, PhD; Neil Aaronson, PhD;

Birthe Krogh Rasmussen, MD, DMSc; and Pernille Bidstrup, PhD

J Natl Compr Canc Netw 2020;18(9):1222-1229

eTable 1: Differences in Characteristics Between Individuals Who Did and Did Not Participate 


\section{eTable 1. Differences in Characteristics Between Individuals Who Did and Did Not Participate}

Patients With Glioma

Cancer-Free Persons

\begin{tabular}{|c|c|c|c|c|c|c|}
\hline & Included in Study & Did Not Participate & $P$ Value & Included in Study & Did Not Participate & $P$ Value \\
\hline Total, $\mathrm{n}$ & 363 & 191 & & 481 & 823 & \\
\hline Sex, n (\%) & & & .74 & & & .27 \\
\hline Male & $220(61)$ & $113(59)$ & & $288(60)$ & $467(57)$ & \\
\hline Female & $143(39)$ & $78(41)$ & & $193(40)$ & $336(43)$ & \\
\hline Age, mean (SD), y & $55.4(13.4)$ & $57(14.4)$ & .22 & $55.2(13.8)$ & $55.5(15.9)$ & .71 \\
\hline Tumor grade, n (\%) & & & .08 & & & \\
\hline 1 and 2 & $82(23)$ & $28(16)$ & & & & \\
\hline 3 & $51(14)$ & $21(12)$ & & & & \\
\hline 4 & $221(63)$ & $126(72)$ & & & & \\
\hline
\end{tabular}

Abbreviation: CES-D, Center for Epidemiologic Studies Depression Scale. 